means of being able to give a definite experimental demonstration).

The Argoment from anmals to Man.

This being the case, evidently the objection might be raised that although this vaccination renders guinea-pigs rabbits, and pigeons very resistant, yet all the same it might be possible that man might escape this law. That, indeed, is not impossible, and-we lay much stress upon the fact-it is by experiment that our method must be proved to be true. It is by direct application and use that we must show ourselves and others the true value of the means extolled. Yet after the experience already acquired we think that there is little probability of a negative result. The difference between a mammal like the rabbit or guinea-pig and a bird like the pigeon from an anatomical and physiological point of view is much greater than between rodents and man; hence when the same law is proved to exist amongst animals so different there is little probability that man is not also subject to it. The symptoms following on inoculation of our vaccines are strictly analogous in all the animals experimented on, including man. The same changes of temperature take place in all the animals. In the animals which have been rendered resistant against cholera, immunity is made evident by the fact that every fresh subcutaneous inoculation, made even with weakened virus, induces a rise of temperature more considerable than the preceding. We hope that the parallel will extend to this modification of the organism which produces the resistance against an infectious agent. But the sole and definitive confirmation of this hope can be obtained only by the application of the method where cholera makes ravages in the human race.

ERRATUM. - In the paper on Haffkine's Method of Vaccination Against Asiatic Cholera in the BRITISH MEDICAL JoURNAL of February 4th, p. 230 col. 2, line 32, the sentence should read, "In the case of the carbolised vaceines this fluid consists of a 0.5 per cent. solution," etc., not 5 per cent., as printed.

\section{SYPHILITIC AFFECTIONS OF THE NERVOUS} SYSTEM.

\section{An Abstract of the Lettsomian Lectures delivered before the Medical Society of London.}

By J. S. BRISTOWE, M.D., LL.D., F.R.C.P., F.R.S., Consulting Physician to St. Thomas's Hospital.

LFCTURE III.

\section{GUMMATA AND PACHYMENINGITIS.}

Di. BRIstowe observed that in the brain true gummata were apt to be surrounded by, and embedded in, cicatricial tissue indistinguishable from the corresponding results of simple non-specific inflammations, and that such cicatricial tracts might furnish the only evidence of syphilitic disease. Such apparently simple inflammatory lesions were probably in many cases, as had been observed in a previous lecture, as truly specific in their origin as gummata themselves. He quoted in full the case of a man, who had contracted syphilis at the age of 28 . His subsequent history was very fally known; he suffered from syphilitic iritis, from painful affections of the limbs ("rheumatism," "sciatica"), and finally had a fit, which left him with severe headache and nausea. He improved for a time, then relapsed, and came under treatment with headache, dimness of vision, and double optic neuritis. He improved greatly under treatment, and remained in fairly good health for two years, when a relapse occurred. He was readmitted, and died about six weeks Tater of gangrene of the lung. The only well-marked lesion observed after death was that over the right occipital lobe the membranes were firmly adherent, and the cortex beneath was soft and yellow. There were no distinct gummata, but there could be little reason to doubt that the meningeal thickening and adhesions were of syphilitic origin. Other cases were quoted in support of the view put forward.

\section{Differegntial Diagnosis.} The difficulty of distinguishing between the symptoms
pr sdi ced by syphilitic disease. of cerebral arteries, by intra- cranial gummata, and by syphilitic inflammation of the membranes or substance of the brain, was dwelt upon. Obstruction of vessels from other causes usually produced sudden paralysis, while the growth of a tumour gave signs of more or less duration. But in all the cases of syphilitic obstruction of arteries Dr. Bristowe had seen the onset of symptoms had been gradual; and, further, in no inconsiderable number of cases of brain tumour no appreciable symptoms were produced until the patient was suddenly seized with an epileptic fit or some other sudden form of illness. Further, arteritis, gummata, and pachymeningitis were often developed simultaneously. Still, if in the course of vague cerebral symptoms a syphilitic patient had some sudden seizure and remained hemiplegic the probabilities were in favour of obstruction of vessels, whereas headache, giddiness, vomiting, optic neuritis, mental perversion or failure, and epileptic attacks pointed to gummata or pachymeningitis, and this would be confirmed by evidence of syphilitic disease of the periosteum, of the skull, or of the ears. Further, gummata and pachymeningitis were especially apt to occur at the base, and to implicate cranial nerves. It was necessary to note that such implication occurred progressively. Syphilitic obstruction of vessels was not limited to the middle cerebrals, as embolic for the most part was, but might involve any of the arteries; consequently softening of the pons or crura might occur and a condition of more or less hemiplegia, with implication of many nerves, be produced closely resembling that which was the consequence of basal gummata. Having regard to the mode of invasion, the character and frequency of the epileptic attacks if present, the sudden or gradual onset of hemiplegia, the grouping and evolution of the paralysis of cranial nerves, a reasonably accurate differential diagnosis might be made in many cases. In the majority, however, no such accuracy was attainable. This, fortunately, was not a matter of great importance in treatment.

Syphilitic Affections of Spinal Cord and Nerpers.

There was abundant proof that within the spinal canal, as within the skull, syphilitic arteritis, gummata, and syphilitic pachymeningitis were all liable to occur. Cases were quoted in which the only possible diagnosis was syphilis. Affection of the spinal nerves outside the spinal canal were probably rare ; the lecturer was unable to quote one from his own experience. Localised anæsthesia, neuralgia, or paralysis occurring in syphilitic persons, and not due to cerebral disease might not unreasonably be suspected to be due to degenerative neuritis of syphilitic origin; but Dr. Bristowe was indisposed to accept the view that symmetrical peripheral neuritis in a syphilitic patient was likely to be distinctly syphilitic.

Syphirtic OpHThalmoplegia.

Dr. Bristowe had never met with total and complete ophthalmoplegia externa excepting in cases of functional nervous disorder.

Conjugate deviation of the eyes, presumably due to some lesion involving the nucleus of origin of one of the sixth nerves in the strand of fibres. passing thence upwards to the opposite hemisphere of the brain, occurred in several of the cases which had been mentioned as actual or suspected cases of cerebral syphilis. In some of these cases the conjugate deviation suddenly ceased after a time, and it was found that the patient could not move the eyes to right or left, while retaining the power of moving them upwards or downwards. This was probably due to the involvement first of the nucleus of the sixth on one side, causing conjugate deviation, and then on the other causing loss of movements in the horizontal plane in both direction. There was no doubt that in syphilis the motor nerves of the eyes were peculiarly prone to suffer, and that not infrequently all of them became involved to a greater or less degree, but the paralysis always remained incomplete and unsymmetrical.

Wr are pleased to learn that Dr. Roberts Bartholow, who some time ago was compelled by illness to resign the chair of Materia Medica and Therapeutics in Jefferson Medical College, is now so completely restored to health that he has re$s$ imed practice in Philadelphia. An article on Cholera from his pen will appear in an early number of the Philadelphia Medical News. 